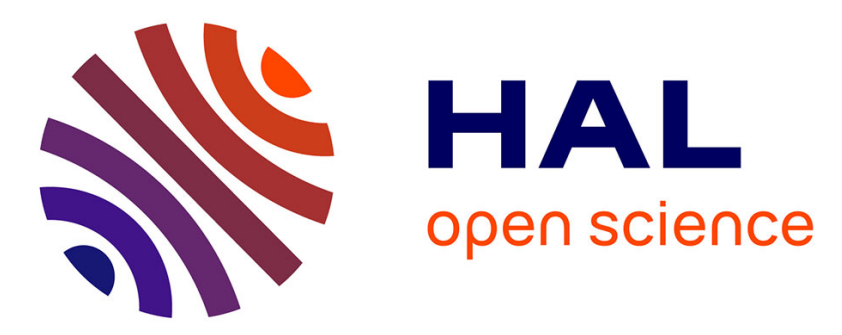

\title{
SERS Correlation Spectroscopy of Silver Aggregates in Colloidal Suspension: Quantitative Sizing Down to a Single Nanoparticle
}

Aude Barbara, Fabien Dubois, Alain Ibanez, Lukas Eng, Pascal Quemerais

\section{- To cite this version:}

Aude Barbara, Fabien Dubois, Alain Ibanez, Lukas Eng, Pascal Quemerais. SERS Correlation Spectroscopy of Silver Aggregates in Colloidal Suspension: Quantitative Sizing Down to a Single Nanoparticle. Journal of Physical Chemistry C, 2014, 118 (31), pp.17922-17931. 10.1021/jp5024444 . hal02001178

\section{HAL Id: hal-02001178 \\ https://hal.science/hal-02001178}

Submitted on 6 Feb 2019

HAL is a multi-disciplinary open access archive for the deposit and dissemination of scientific research documents, whether they are published or not. The documents may come from teaching and research institutions in France or abroad, or from public or private research centers.
L'archive ouverte pluridisciplinaire HAL, est destinée au dépôt et à la diffusion de documents scientifiques de niveau recherche, publiés ou non, émanant des établissements d'enseignement et de recherche français ou étrangers, des laboratoires publics ou privés. 


\title{
SERS Correlation Spectroscopy of Silver Aggregates
}

\section{in Colloidal Suspension: Quantitative Sizing Down to a Single Nanoparticle}

\author{
Aude Barbara, ${ }^{*, \dagger, \dagger}$ Fabien Dubois, ${ }^{\dagger}$ Alain Ibanez, ${ }^{\dagger}$ Lukas M. Eng,,${ }^{\dagger}$ and Pascal \\ Quémerais $^{\dagger}$ \\ Univ. Grenoble Alpes, Inst NEEL, F-38042 Grenoble, France \\ CNRS, Inst NEEL, F-38042 Grenoble, France, and Institut für Angewandte Photophysik, \\ Technische Universität Dresden, 01062 Dresden, Germany \\ E-mail: aude.barbara@neel.cnrs.fr
}

*To whom correspondence should be addressed

$\dagger$ Univ. Grenoble Alpes, Inst NEEL, F-38042 Grenoble, France

CNRS, Inst NEEL, F-38042 Grenoble, France

†nstitut für Angewandte Photophysik, Technische Universität Dresden, 01062 Dresden, Germany 


\begin{abstract}
The slow flocculation of silver nanoparticle (NP) aggregates obtained through para-mercaptobenzoic acid (MBA) linker molecules was analyzed by surface enhanced Raman scattering (SERS) correlation spectroscopy. We demonstrate that our experimental set-up, together with the quantitative exploitation of the SERS intensity auto-correlation functions (ACFs) measured from the MBA molecules, enables to determine quantitatively and in situ the size of the SERS active clusters. Moreover, we show that it is possible to characterize the different states of aggregation during cluster formation simply by measuring the size of such silver aggregates. This has been accurately performed for both larger-size aggregates down to very few NPs only. We finally show that our approach is also sensitive to detect and analyze the SERS signal obtained from MBA molecules sitting on non-aggregated NPs. These experiments pave the way to the in situ characterization of functionalized metal NPs as well as the in situ monitoring of NP aggregation, in conjunction with deriving the optical properties of the as-formed clusters.
\end{abstract}

Keywords: SERS-active clusters sizing, autocorrelation functions, hydrodynamic properties of aggregates, confocal microscopy

\title{
Introduction
}

Noble metal colloids exhibit rich optical properties that are predominantly governed by the ability of metallic nanoparticles (NPs) to sustain localized surface plasmon modes. Such resonances can be excited by an incident electromagnetic wave in the visible spectral range for $\mathrm{Au}$ and $\mathrm{Ag}$ NPs, giving rise to resonantly enhanced absorption and emission properties ${ }^{1,2}$ as well as field enhancement at the surface of these NPs. ${ }^{3,4}$ The latter constitutes a key element in surface enhanced Raman scattering (SERS). In 1981, Aravind et al. ${ }^{5}$ showed in a theoretical work that the electromagnetic interaction of two metal spheres approached to a close distance, much smaller than the exciting wavelength, may cause a substantial enhancement of the local electric field in the confined space between the two spheres. The strength of the field enhancement rapidly decreases with increasing particle separation. Also the field enhancement may be optimized by adequately 
adjusting the incident light beam, being polarized along the dimer axes as well as hitting one of the plasmon resonances of the coupled sphere system. Under these conditions and for interparticle distances of about $1 \mathrm{~nm}$, very large Raman enhancements on the order of $10^{10}$ have been predicted for molecules located within the so-called hot-spot region between the spheres. ${ }^{5,6}$ Adding more spheres to form larger aggregates rapidly increases the complexity of the system, hence posing a non-trivial task to associate the measured spectral response to the information solely given through the resonant behavior of each individual NP. In particular when fractal aggregates are formed by self-assembly or through flocculation of NPs, resonances might appear as reported for commensurate arrangements of dipoles, ${ }^{7}$ that do not exist for periodic or ordered systems. Such aggregates may support giant local field enhancements ${ }^{8}$ possibly promoting single molecular Raman detection. ${ }^{9,10}$ The advent of single molecule SERS has led to the development of various experimental techniques for the ultra-sensitive and selective detection of molecules with a manifold of applications in physics, chemistry and biology. ${ }^{4}$ More recently, metal NPs are discussed also as the very prospective candidates for in vivo medical applications, such as intra-cellular imaging ${ }^{11}$ or in-body drug delivery. ${ }^{12}$ Other applications based on metal NPs are also under investigation, for instance in the field of nanoscale thermally-activated processes such as nano-catalysis ${ }^{13-15}$ or photo-thermal therapy. ${ }^{16,17}$

Over the last few decades, intense research work has been dedicated to such colloidal solutions with the clear goal of understanding and possibly controlling their behavior for nano-device applications. However, determining the correlation between the geometry of isolated or coagulated NPs and their activity, as well as inspecting aggregation processes per se, still poses a highly challenging task. To date, we may distinguish between two basic approaches: The first class of "experiments" concentrates on objects that are immobilized on a substrate surface. This allows for analyzing the properties of the objects through dedicated microscopical techniques such as transmission electron microscopy or atomic force microscopy, in correlation with dark-field spectroscopy, SERS and/or absorbance measurements. ${ }^{18}$ Using these methods allows quantifying both the averaged particle-ensemble and single-object responses, hence promoting a direct link between 
the images of the object and its optical properties. The second set of experiments focuses on socalled solution-based approaches, where particle diffusion is accessed via the analysis of the temporal fluctuations of a specific spectral physical quantity. The latter may constitute in monitoring the free diffusion of NPs through a finite optical volume maintained by a confocal microscope, ${ }^{19,20}$ by flow-cytometry ${ }^{21}$ or dynamic imaging ${ }^{22,23}$ techniques, or as recently proposed also by time-offlight photo-spectrometry. ${ }^{24}$ This second class of tools allows even for an in situ analysis, showing clear advantage whenever the solvent itself may affect the NPs' activity. Moreover, a direct correlation of the measured spectral quantity to the size of the object can be established. Some of these works propose the SERS signal to be selected as the spectral physical quantity, for instance to characterize the brightness and uniformity of SERS-active NPs by a flow spectrometry approach ${ }^{21}$ or to determine the diffusion coefficient of the SERS-active aggregates by the dynamic imaging of the SERS signal. ${ }^{25}$

In the present paper, we propose to characterize colloidal suspensions of aggregates of silver NPs by SERS correlation spectroscopy. This method, analogously to fluorescence correlation spectroscopy $^{26}$ (FCS), aims at measuring the temporal fluctuations of the SERS signal of molecules adsorbed on clusters of NPs as they diffuse through a confocal volume, in order to access their diffusion coefficient. Our goal is twofold: First, we aim at demonstrating the pertinence of the approach for the quantitative and in situ sizing of the SERS-active clusters. Secondly we ultimately show the fingerprint of the aggregation process through the identification of self-assembled NPs clusters in different stages of aggregation. The very first SERS correlation spectroscopy measurements were reported already in 2001 by Eggeling et al. ${ }^{27}$ who showed that such technique enabled to select, out of an heterogeneous solution, a single molecule/particle ensemble and identify its anisotropy, brightness and rotational diffusion. Since then, only very few works reporting on SERS correlation spectroscopy evolved, i.e. only two articles are published ${ }^{28,29}$ to the best of our knowledge. The authors of these works used the same technique as ours in order to measure the rotational diffusion coefficient of Ag NP dimers, and mostly focused on the quantification of the enhancement factor of the SERS effect confined in the metal cavities. However, no convincing 
in situ sizing and identification of cluster type was achieved. Recently, we have shown ${ }^{30}$ that our experimental set-up, specifically optimized for Raman and SERS correlation spectroscopy, possesses a very high sensitivity to realize Raman correlation spectroscopy for signal strengths that are several orders of magnitude weaker than those usually published. Here, we take advantage of this high sensitivity in pushing the precise detection and identification of SERS clusters down to the non-aggregated NPs level, known to produce low SERS activities.

As a first demonstration of the high potential of in situ SERS correlation spectroscopy, we investigated model systems of colloidal suspensions made up from silver NP aggregates in which the aggregation of NPs is induced by para-mercaptobenzoic acid (MBA) molecules in ethanol. The MBA molecules adsorb to the NPs and act both as molecular linkers between NPs as well as SERS probes. MBA molecules thus are actively placed in the hot spot regions between clusters. Standard absorbance measurements were performed in order to check on the colloidal stability of the aggregate suspension while Raman spectra validated their SERS activity. The temporal fluctuations of the SERS signal were then measured and analyzed to quantify the size of the Ag aggregates by deriving the auto-correlation functions (ACFs) of the SERS intensity. As a result, we were able to measure the size and diffusion coefficient of both larger clusters, that give rise to time-integrated SERS intensities of a few kcounts per second, but equally of very small aggregates composed of a very few number of NPs that present time-integrated SERS intensities $\sim 5$ times smaller. The SERS intensities are proportional to the enhancement factors and concentration of the aggregates and to the effective measuring time of the SERS intensities. The latter effective time is proportional to the characteristic time of diffusion through the observation volume, that scales as $\ell$, and to the characteristic time of rotational of the aggregate that scales as $\ell^{3}$, where $\ell$ is the length of the aggregate. The variation of the measuring time as a function of the aggregates sizes may thus account for the observed behavior of the SERS intensities, even if this effect is partially balanced by the variation of the aggregates concentration which decreases as the clusters become larger. Finally, the SERS enhancement factors of the aggregates are not known here but they are considered to play no significant role in the modification of the SERS intensities as it was reported that they do 
not correlate with the aggregation state. ${ }^{31}$ At the end of the paper, we finally give clear evidence for measuring also the diffusion coefficient of non-aggregated NPs by this method, i.e of single NPs. These results pave the way to a step-by-step characterization of aggregation processes and the characterization of single NP tags with high chemical selectivity.

\section{SERS correlation spectroscopy}

Principle. In SERS correlation spectroscopy experiments, a solution containing nanoparticles that are covered by SERS probes molecules (here MBA) is illuminated in a confocal microscope that confines the observation volume to typically a few fL. In the present case, the particles are either single silver NPs or NP aggregates. The diffusive motion of aggregates into and out of the observation volume induces temporal fluctuations of the measured SERS signal. The characteristic time of these fluctuations is called the translational diffusion time and is denoted $\tau_{D}$. Its value is directly linked to the time spent by the particle within the observation volume. Knowing $\tau_{D}$ furthermore enables quantifying the translational diffusion coefficient $D_{D}$ of the particles. When the incident light is polarized and when the scattering of the molecules is anisotropic, the particle rotation provokes an additional temporal fluctuation due to the misalignment between polarization orientation and the main axes of the scattering tensor. Accordingly, a characteristic time $\tau_{R}$ related to the rotational diffusion coefficient $D_{R}$ of the particles may be deduced. Note that both $\tau_{R}$ and $D_{R}$ are not relevant for isotropic systems.

Autocorrelation functions. In order to access the above-mentioned characteristics, the timedependent intensity $I(t)$ of the chosen SERS band is measured and statistically analyzed by deriving its normalized autocorrelation function $G(t)$. Since $\tau_{D}$ and $\tau_{R}$ proceed on very different time scales, their respective contributions to the ACF can be separated ${ }^{32,33}$ so that:

$$
G(t)=\frac{\langle I(\tau) I(t+\tau)\rangle}{\langle I(\tau)\rangle^{2}}=G_{D}(t) \cdot G_{R}(t)
$$


where $G_{D}(t)$ and $G_{R}(t)$ are the translational and rotational ACF, respectively, allowing to quantify $\tau_{D}$ and $\tau_{R}$ through monitoring their temporal decay. The expressions of $G_{D}(t)$ and $G_{R}(t)$ are obtained using the theory developed for $\mathrm{FCS}^{34,35}$ and for dynamic light scattering (DLS) in small volumes, ${ }^{36}$ as well as accounting for the effective intensity profile within the confocal volume to be an anisotropic Gaussian function of the form $I(x, y, z)=I_{0} e^{-2\left(x^{2}+y^{2}\right) / r_{0}^{2}-2 z^{2} / z_{0}^{2}}$, where $I_{0}$ is the incident laser power intensity and $r_{0}$ and $z_{0}$ are the radii of the profile in the (x,y)-plane and along the $\mathrm{z}$ direction, respectively (note that the $\mathrm{z}$ direction is aligned parallel to incident $\mathrm{k}$-vector). The ACF may then be expressed as:

$$
G(t)=1+\frac{c^{2}}{\langle N\rangle} \frac{1}{\left(1+t / \tau_{D}\right)\left(1+t / w^{2} \tau_{D}\right)^{0.5}} \cdot\left(1+R e^{-t / \tau_{R}}\right)
$$

where $\tau_{D}=r_{0}^{2} / 4 D_{D}$ and $\tau_{R}=1 / 6 D_{R} . w=z_{0} / r_{0}$ is the shape parameter and $\langle N\rangle$ is the average number of particles in the confocal volume while $c=1-\left\langle I_{B}\right\rangle /\langle I\rangle$ is the background correction factor with $\langle I\rangle$ being the averaged total intensity and $\left\langle I_{B}\right\rangle$ that of the background. ${ }^{37}$ Finally, the coefficient $R$ denotes the ratio of rotational to translational amplitude in the ACF, and depends on the geometry of the experiment and on the degree of anisotropy as given by the scatterers. In the absence of polarized light or for symmetric scattering, $R=0$ and $G(t)=G_{D}(t)$ since particle rotation does not influence the measured intensities.

Particle sizing. The characteristic times $\tau_{D}$ and $\tau_{R}$ are extracted from the experimental ACFs by fitting them to Eq. 2. The value $r_{0}=445 \mathrm{~nm}$ needed to access $D_{D}$ from $\tau_{D}$ was previously determined by calibration measurements, ${ }^{30}$ while $D_{R}$ is directly accessed from $\tau_{R}$. Once the two diffusion coefficients $D_{D}$ and $D_{R}$ are numbered, the size and shape of the particles can be determined, provided that an appropriate theoretical model can be applied. In the present paper, we use three different models:

- Model (I) is the simplest and most commonly used one: it models the diffusing aggregate by means of a spherical particle of effective hydrodynamic radius $r_{H}$, which is related to the 
diffusion coefficients via the Stokes-Einstein equations ${ }^{38-40}$ through:

$$
\operatorname{Model}(\mathrm{I})\left\{\begin{array}{c}
D_{D}=k_{B} T / 6 \pi \eta r_{H} \\
D_{R}=k_{B} T / 8 \pi \eta r_{H}^{3}
\end{array}\right.
$$

where $k_{B}$ is the Boltzmann constant, $\mathrm{T}$ the temperature, and $\eta$ the viscosity of the solvent. The temperature $T=24^{\circ} \mathrm{C}$ was estimated by calibration measurements ${ }^{30}$ while $\eta(T=$ $\left.24^{\circ} \mathrm{C}\right)=0.911 \times 10^{-3} \mathrm{~kg} \cdot \mathrm{m}^{-1} \cdot \mathrm{s}^{-1}$ is a tabulated value. In this model, the effective radius $r_{H}$ corresponds to the radius of a sphere that produces the same hydrodynamic drag as does the aggregate under inspection.

In the case of close-packed NPs forming quasi-spherical aggregates, $r_{H}$ is directly related to the cluster radius, and the model is very accurate. However, in many other cases, aggregates of arbitrary shape may be present (i.e. from chain-like clusters to self-similar fractal geometries); here, a more sophisticated approach is needed in order to relate the actual size and shape of the diffusing object to its effective hydrodynamic radius.

- Model (II): The second approach, the so-called bead model, was first proposed by Bloomfield ${ }^{41}$ and bases on the Kirkwood-Riseman theory. ${ }^{42}$ Arbitrarily shaped aggregates are modeled through spherical beads of either identical or variable sizes in such a way that they best accommodate with the effective geometry of the aggregate. This model has been successfully applied to linear (flexible or rod-like) chains of NPs. ${ }^{43,44}$ Also, the bead model is suitable to predict the size dependence of the hydrodynamic particles in a fractal aggregate on its radius of gyration and its fractal dimension. ${ }^{45}$ In the present case we are dealing with small aggregates composed of a few mono-disperse NPs only (see section 'experimental methods'). We thus may apply the bead model in such a way as to consider our aggregates to be made-up from chains of $n$ identical beads with hydrodynamic radius $r_{H}$. ${ }^{43,44}$

- Model (III): Finally, the third model of interest is the cylinder model, ${ }^{46}$ where aggregates containing a few number of NPs only are modeled by a cylinder of radius $r_{H}$ and length 
$L=2 r_{H} \times n$. Here $n$ is the number of NPs contained in the aggregate, and $r_{H}$ is the radius of the NPs.

In both models (II) and (III), the diffusion and rotational coefficients $D_{D}(n)$ and $D_{R}(n)$ of aggregates composed of $n$ NPs are calculated as a function of $n$ and $r_{H}$. However, in order to generalize these results, it is appropriate to express them in a normalized way, i.e. independently of the size of the isolated NPs, by introducing the diffusion and rotational coefficients $D_{D}^{0}$ and $D_{R}^{0}$ of the single NP defined as:

$$
\begin{array}{lll}
D_{D}^{(i)}(n)=\alpha^{(i)}(n) D_{D}^{0} & \text { with } & D_{D}^{0}=k_{B} T / 6 \pi \eta r_{H} \\
D_{R}^{(i)}(n)=\beta^{(i)}(n) D_{R}^{0} & \text { with } & D_{R}^{0}=k_{B} T / 8 \pi \eta r_{H}^{3}
\end{array}
$$

where $i \in\{I I, I I I\}$. The coefficients $\left\{\alpha^{(i)}(n), \beta^{(i)}(n)\right\}$ are reported in Table 1 . Note that for the Table 1: Values of the coefficients $\alpha(n)$ and $\beta(n)$ for the two models: (II) (beads model) and (III) (cylinder) as a function of the number $n$ of single NPs contained in a small aggregate. These coefficients are defined as $\alpha(n)=D_{D}(n) / D_{D}^{0}$ and $\beta(n)=D_{R}(n) / D_{R}^{0}$, where $D_{D}^{0}=k_{B} T / 6 \pi \eta r_{H}$ and $D_{R}^{0}=k_{B} T / 8 \pi \eta r_{H}^{3}$. The numerical values are extracted from Refs.

\begin{tabular}{|c|c|c|c|c|}
\hline & \multicolumn{2}{|c|}{ Model (II) } & \multicolumn{2}{|c|}{ Model (III) } \\
\hline$n$ & $\alpha^{(I I)}(n)$ & $\overline{\beta^{(I I)}(n)}$ & $\alpha^{(I I I)}(n)$ & $\beta^{(I I I)}(n)$ \\
\hline 2 & 0.72 & 0.27 & 0.63 & 0.18 \\
\hline 3 & 0.58 & 0.11 & 0.53 & 0.08 \\
\hline 4 & 0.49 & 0.06 & 0.46 & 0.045 \\
\hline 5 & 0.42 & 0.04 & 0.40 & 0.03 \\
\hline
\end{tabular}
[42,43] for Model (II) and Ref. [45] for Model (III).

rest of this paper whenever only one single correlation time $\left(\tau_{D}\right)$ is measured for a given type of aggregate, we assume this aggregate to be essentially spherical in shape, hence allowing to apply Model (I). In case of aggregates that contain only a few numbers $(n<5)$ of NPs, the two diffusion times $\tau_{D}$ and $\tau_{R}$ can be measured (see section 'results'), giving access to the $D_{D}(n)$ and $D_{R}(n)$ values. For both these values and for each possible $n, r_{H}$ may be estimated as $r_{H}=\frac{\alpha(n)}{D_{D}(n)} \frac{k_{B} T}{6 \pi \eta}$ and $r_{H}=\sqrt[3]{\frac{\beta(n)}{D_{R}(n)} \frac{k_{B} T}{8 \pi \eta}}$. The number $n$ of NPs actually contained in the measured aggregate is then determined through matching the $r_{H}$ values as derived from $D_{D}$ and $D_{R}$. Then, and only then, 
$r_{H}$ matches also to the nominal value of non-aggregated NPs, i.e. $r_{H}^{0}=10 \pm 2 \mathrm{~nm}$ (see section 'experimental methods').

\section{Experimental methods}

Starting materials. Silver nitrate $\left(\mathrm{AgNO}_{3}, \mathrm{ACS}\right.$ reagent, $\left.99.5 \%\right)$, sodium borohydride $\left(\mathrm{NaBH}_{4}\right.$, purum p.a., > 96\%), para-mercaptobenzoic acid (MBA, 99\%) and sodium citrate tribasic dihydrate (sodium citrate, ACS reagent $>99 \%$ ) were purchased from Aldrich. Ethanol $95 \%$ was obtained from Sodipro. High-purity water with a resistivity of $18 \mathrm{M} \Omega \mathrm{cm}$ was used (Millipore ${ }^{\complement}$ ) to prepare silver nanoparticles. Filters (100 nm pores) were purchased from Sartorius.

Nanoparticle synthesis. For the preparation of the initial solution of silver NPs we used a modified Creighton method ${ }^{47-49}$ consisting in an aqueous reduction of $\mathrm{AgNO}_{3}$ with $\mathrm{NaBH}_{4}$ followed by adding sodium citrate as a stabilizing reagent. We used de-ionized water to prepare a $1 \mathrm{mM}$ aqueous solution of $\mathrm{AgNO}_{3}$ and a $2 \mathrm{mM}$ aqueous solution of $\mathrm{NaBH}_{4}$. We then added drop-by-drop (6 $\mathrm{mL} / \mathrm{min}$ ) $20 \mathrm{~mL}$ of the $\mathrm{AgNO}_{3}$ solution cooled to $10^{\circ} \mathrm{C}$, to $30 \mathrm{~mL}$ of the $\mathrm{NaBH}_{4}$ solution cooled to $5^{\circ} \mathrm{C}$, all kept in an ice bath under permanent stirring. Ag NP formation showed up by a clear color change of the solution from transparent to lemon yellow. After fully adding the $\mathrm{AgNO}_{3}$ solution, stirring was stopped and $5 \mathrm{~mL}$ of a $13.6 \mathrm{mM}$ aqueous solution of sodium citrate (corresponding to a [sodium citrate $] /[\mathrm{Ag}]=3.4)$ was added to stabilize the colloidal suspension. Finally, the solution was filtered through a 100-nm-pore paper to remove small hypothetical aggregates present after the synthesis. This method yields monodisperse silver NPs with an average diameter of $17 \pm 5$ nm determined by statistical measurements of particles imaged on a Carl Zeiss Ultra SEM. An example is presented in Figure 1A. In solution, the hydrodynamic diameter $d_{H}$ of the particles is larger due to the electric layer surrounding the particles. Its mean value $\bar{d}_{H}=20 \mathrm{~nm}$ was obtained by DLS measurements performed on a Zetasizer Nano Zs, Malvern company. An example of particle sizing realized by DLS measurements using our optical set-up (see next section) is shown in

figure $1 \mathrm{~B}$. The characteristic time $\tau_{D}=2.3 \mathrm{~ms}$ correspond to an hydrodynamic radius $r_{H}^{0}=11 \mathrm{~nm}$ in agreement with the values of the particle sizes and and size distribution. The concentration of 
NPs was roughly estimated by calculating the ratio of the total mass of silver to the mass of a 17 nm-diameter silver sphere thus by neglecting the removed hypothetical aggregates. This yields an upper-bound estimate of $\sim 1.5 \times 10^{12} \mathrm{NPs} / \mathrm{mL}$.

NP aggregation. The flocculation of silver NPs covered by MBA molecules may be induced at different strategies; in most cases, the MBA is either adsorbed to NPs prior to ${ }^{50}$ or after $^{28}$ coag- $^{-}$ ulation of NPs that was induced by a salt or a bifunctional molecule. In our case the aggregation of the NPs was obtained solely by mixing $600 \mu \mathrm{L}$ of the initial aqueous suspension of Ag NPs with different volumes of MBA ethanol solutions, in order to obtain a final concentration of MBA in the samples ranging from $1 \mathrm{mM}$ to $10^{-3} \mathrm{mM}$. MBA adsorbs onto the silver NPs, presumably in a uniform way, and link the NPs. MBA thus is used here as both the molecular linker between NPs and as the SERS probe. Successful aggregation was indicated through the color change and analyzed in the spectral range from 350-800 nm by absorption spectroscopy (Shimadzu UV-3101 $\mathrm{PC})$.

Optical set-up. The optical set-up used to record Raman spectra and to perform SERS correlation spectroscopy measurements is detailed in ref. ${ }^{30}$ and is sketched in Figure 2. It consists of an optically inverted Zeiss microscope (Axio-vert 200) equipped with an oil-immersion objective $(1.45 \mathrm{NA}, 100 \times)$. Samples are illuminated by a linearly polarized laser source of wavelength $\lambda_{0}$ $=532 \mathrm{~nm}$ with an incident power of $P_{0}=50 \mathrm{~mW}$. The backscattered light (Rayleigh and Raman) is collected by the same objective and is focused by a tube lens onto a $300 \mu$ m-diameter pinhole placed in the backfocal plane. The latter selects an observation volume $V_{c} \sim 1.6 \times 10^{-12} \mathrm{~cm}^{3}$. $^{30}$ A Notch filter with an optical density of 6.0 is placed behind the pinhole to suppress the Rayleigh scattered light, while Raman photons are transmitted and focused at the entrance slit of a Raman spectrometer (Spectro Solar TII, focal length $750 \mathrm{~mm}$ ). The spectrometer permits either to record SERS spectra by using a 75-groove/mm grating and a cooled CCD camera (Andor DU 440), or to select the photons of a chosen SERS band to exit the spectrometer through a 0.5 -mm-wide slit. In this configuration, the spectral bandwidth of the collected Raman line is $9 \mathrm{~nm}$. That outgoing beam is collimated onto a $20 \times$ objective which focuses the beam onto an avalanche photodiode 
(APD Perkin Elmer, SPCM-AQR-15) and is coupled to a photon correlator (Ciprian). The latter measures the time interval between two incoming pulses to reconstruct the time-dependent intensity $I_{\lambda}(\mathrm{t})$ of SERS photons at the design wavelength $\lambda$ and derives the ACF $G(t)$ of $I_{\lambda}(\mathrm{t})$. DLS measurements can also be realized by removing the Notch filter and selecting the Rayleigh wavelength as an output of the spectrometer instead of the wavelength of a SERS band.

\section{Results and discussion}

Nanoparticle aggregation process and colloidal stability. When adding MBA to the Ag NPs solution its color gradually changes indicating NP aggregation that then stabilizes after approximately 20 minutes. Two mechanisms are reported in the literature that account for this coagulation process:

- (i) aggregation due to MBA molecules which are bound to the NP surfaces both through sulfur atoms and $\mathrm{COO}^{-}$groups; ${ }^{51}$ the sulfur atoms bind to the surface in a coordinative bonding while carboxylic groups interact with the silver substrate to form carboxylates. ${ }^{52}$

- (ii) aggregation due to van der Waals forces since electrostatic repulsion between NPs is reduced; this preferentially occurs whenever the thickness of their electrical double layer is reduced through counter ions present in the solution $\left(\mathrm{H}^{+}\right.$or $\left.\mathrm{Na}^{+}\right) .{ }^{50}$

The kinetics of aggregation was monitored by recording absorption spectra in 2-minutes intervals. Figure 3A shows an example of the evolution of the extinction spectra measured from a silver NP solution in the presence of $1.58 \mathrm{mM}$ MBA. The initial silver solution shows clear absorption at $390 \mathrm{~nm}$ as expected for spherical NPs with a radius $r_{H}^{0}=10 \mathrm{~nm} .{ }^{2}$ One minute after adding MBA molecules, the spectrum is still dominated by that extinction band, however, with its center peak-position now slightly red-shifted to $\lambda=405 \mathrm{~nm}$. This effect can be readily attributed to the presence of MBA that modifies the dielectric constant of the solvent near the NP surface and hence leads to the observed plasmonic red shift. ${ }^{53}$ The position of this band is not further changing while 
its intensity decreases considerably as a function of time, indicating the reduction of the number of isolated NPs. At the same time, cluster formation sets in showing up in a second and red-shifted absorption peak that manifests the multipolar plasmonic resonances of aggregates. During the initial stages of aggregation, the second band grows in intensity and shifts towards larger wavelengths, inducing a color change of the solution. A little later, stabilization is observed in both the extinction spectra and the color of the solution. At even longer times (Figure 3B), both the spectral shifting and intensity-decrease of the two extinction bands proceed very slowly only; these changes are assessed to both the possible sedimentation and/or the partial adsorption of NPs/NP-aggregates to the walls of the cuvette. A similar behavior is observed for other MBA concentrations in solution, i.e. from $10^{-2}$ to $1 \mathrm{mM}$. When stabilized, the color of these different solutions may range from orange to brownish-red (with increasing MBA concentration) while the position of the second absorption band varies from $535 \mathrm{~nm}$ to $595 \mathrm{~nm}$. A yellow color (as was the case for purely NP-loaded solutions) was observed only when keeping the MBA concentration low, i.e. at $10^{-3} \mathrm{mM}$. Also, no second absorption peak popped up in this case which is a clear sign of inhibited NP aggregation. The obtained colloidal suspensions were stable for several hours after preparation, thus allowing for their stable inspection by Raman and SERS correlation spectroscopy.

Raman Spectra. Figure 4A presents a typical Raman spectrum (acquisition time $30 \mathrm{~s}$ ) as obtained on a colloidal suspension of NPs aggregated with MBA. Successful MBA adsorption to NPs is confirmed by registering the two strong SERS bands at 1085 and $1590 \mathrm{~cm}^{-1}$, assigned to $v_{12}$ and $v_{8 a}$ aromatic ring vibrations, respectively, ${ }^{51,52}$ and a band at $1430 \mathrm{~cm}^{-1}$ which is attributed to the $v_{s}\left(-\mathrm{COO}^{-}\right)$vibration of the carboxylate $-\mathrm{COO}^{-}$groups on the metal surface. ${ }^{51,52}$ Moreover, no bands are observed at $1710 \mathrm{~cm}^{-1}$ where the $\mathrm{C}=\mathrm{O}$ stretching vibrations of non-dissociated $\mathrm{COOH}$ groups are expected, nor at $2580 \mathrm{~cm}^{-1}$ where the $v(\mathrm{SH})$ vibrations of the $-\mathrm{SH}$ groups are expected. ${ }^{51}$ These features support the aggregation scenario as described above upon which MBA binds to the NPs through dissociated -SH and - $\mathrm{COOH}$ groups. In addition to the MBA SERS signal, the Raman spectrum also exhibits the Raman signature of the solvent, i.e. the broad band of water seen around $3400 \mathrm{~cm}^{-1}$ and that of ethanol at $2935 \mathrm{~cm}^{-1}$. 
Results of SERS correlation spectroscopy. Temporal fluctuations of the SERS intensity. The Raman spectrum presented in Figure 4A was acquired over a much longer time span than is the aggregates' diffusion time. As a consequence, the temporal fluctuations of the SERS signal are averaged and the measured SERS intensities are proportional to the mean number of SERS-active clusters in the observation volume i.e. proportional to their concentration. Lowering the averaging time (typically to a few $\tau_{D}$ ), however, allows direct access to the real-time SERS signal: Figure 4B displays strong SERS intensities indicating that at least one SERS-active cluster was present in the observation volume during the measurement time of $100 \mathrm{~ms}$. Conversely, only the Raman signal from the solvent is observed in Figure 4C, since either no SERS-active cluster or no cluster at all, diffused through the observation volume while measuring. In SERS correlation spectroscopy, we exactly acquire and analyze these temporal intensity fluctuations in order to access the size of the SERS-active clusters. In the work presented here, we selected the strongest MBA band at 1590 $\mathrm{cm}^{-1}$ for quantifying the correlation and to calculate the ACF. Notably, in the absence of such a SERS signal, we access the background intensity only.

Identifying SERS-active clusters at different stages of aggregation. The formation of clusters results from the self-assembly of NPs that stick together as they encounter each other due to diffusive motion. In the very early stages of aggregation, the solution contains essentially monomers that gradually coagulate to dimers, trimers, and further on, to more complex structures with potentially fractal dimension. ${ }^{54,55}$ Depending on these aggregation stages, three different scenarios may be distinguished by the ACF:

- (i) the case where MBA molecules are adsorbed to the Ag NPs but no aggregation of NPs has occurred yet

- (ii) the stage where the MBA molecules have induced the formation of larger clusters, and

- (iii) the intermediate situation where small aggregates (dimers, trimers,...) have been formed.

In the first case, ACF probes the diffusion of symmetrical spherical NPs [see Model (I)] on which the MBA molecules are assumed to adsorb uniformly. Under such circumstances, the Raman scat- 
tering is isotropic with respect to the incident light polarization, and the ACF will only reveal the translational diffusion time $\tau_{D}$ of individual NPs. Its value should thus correspond to the hydrodynamic radius $r_{H}^{0}=10 \mathrm{~nm}$ of the NPs within the silver solution.

As soon as a dimer is formed the context changes drastically since the Raman scattering of the molecules in-between the two NPs is strongly enhanced and becomes polarization-dependent. ${ }^{5}$ This leads to a rotational contribution in the ACFs whose amplitude depends on the geometry of the set-up. In that case, Models (II) and (III) can be applied appropriately to identify the dimers. This is also true for the intermediate stage of aggregation when dealing with a few numbers of NPs. Here the expected ACFs must reflect both the characteristic translation and rotation times $\tau_{D}$ and $\tau_{R}$ that all quantifying $r_{H}$ which is expected to be close to $r_{H}^{0}=10 \mathrm{~nm}$. Also the number of NPs $n$ in our aggregate can be specified. Note that the rotational contribution to the ACF signal is maximized when dimers are present only, since the scattering tensor then has only one preferential scattering direction. When more NPs are added, more scattering directions appear and hence the rotational contribution of the ACF decreases. The reduced amplitude of the rotational ACF signal thus is accompanied by the growth of the aggregates ${ }^{29}$ and an increase of $\tau_{D}$ and $\tau_{R}$.

Finally, as NPs continue to aggregate, larger clusters composed of a large assembly of NPs in different directions are formed. The number of the preferential orientation of scattering increases and Raman scattering becomes isotropic again on average. Consequently, the ACFs expected in the case of large clusters should also reveal the translational diffusion time $\tau_{D}$ only, as is the case for the single NPs; nevertheless, the hydrodynamic radius must approach $r_{H} \gg 10 \mathrm{~nm}$, as discussed for a fully spherically isotropic case in the model (I). In order to demonstrate the potential of our experiment in terms of SERS correlation spectroscopy, we checked on our ability to access these different types of ACFs and to perform a quantitative characterization of the different aggregation states of NPs, from large clusters down to the very early stages of aggregation, possibly down to the scale of a single particle. Figure 5 presents four experimental ACFs (dotted) that were obtained when measuring solutions with different MBA concentrations ranging from $1 \mathrm{mM}$ to $10^{-3} \mathrm{mM}$. Each measured ACF can be excellently reproduced by the calculated ACF behavior (lines) using 
Eq. 2. As we will see, the parameters used for these calculations are in full agreement with the theoretical predictions described here above and allow to attributing the different ACF to different types of clusters. In Figure 5A, a very high quality ACF is observed: the intercept value $G(0)-1$ is large, a flat and almost noise-free plateau is observed at short times and the experimental timedecay is in very good agreement with the expected $G(t) \sim t^{-3 / 2}$ law for longer times. In this ACF, only the translational contribution is measured with a characteristic time $\tau_{D}=58 \mathrm{~ms}$ corresponding to a radius $r_{H}=280 \gg 10 \mathrm{~nm}$ by applying model (I). This value is much larger than the one found for the monomer. Since also no rotational contribution is observed, this ACF exemplifies the diffusion of a large cluster having a sufficiently large number of scatterers to be isotropic on average. Finally, note that the value $G(0)-1=4$ in Figure 5A, which implies that $\langle N\rangle<1$. Hence, no SERS-active cluster must have entered the observation volume during part of the acquisition time. This result is in accordance with the observation of intermittent SERS intensity bursts presented in Figures 3B and 3C, and obtained from a comparable solution, i.e. with a MBA concentration of $\sim 1 \mathrm{mM}$. This yields additional information on the validity of the measured ACF.

In Figures 5B and 5C, two time-decays are observed in the measured ACFs (dotted) and can be correctly reproduced by ACF calculations using Eq. 2 when including rotational contributions. The fit to our experimental data leads to $\tau_{D}=4.2 \mathrm{~ms}, \tau_{R}=20 \mu \mathrm{s}$, and $R=0.75$ (for Figure 5B), while $\tau_{D}=3.2 \mathrm{~ms}, \tau_{R}=5 \mu \mathrm{s}$, and $R=1.8$ (for Figure 5C). If we apply the spherical model [model (I), eq.(3)] to the diffusion time $\tau_{D}$, we obtain $r_{H} \approx 20.2 \mathrm{~nm}$ and $r_{H} \approx 15.4 \mathrm{~nm}$ for Figures 5B and $5 \mathrm{C}$, respectively. Now, from the expression of the rotational time $\tau_{R}$ of the same model, the values obtained become $r_{H}=27.8 \mathrm{~nm}$ and $r_{H}=17.5 \mathrm{~nm}$, for Figures 5B and 5C, respectively. Applying the spherical model hence is clearly inconsistent since $r_{H}$ deduced from $\tau_{D}$ and $\tau_{R}$ takes on two different values for the two cases (20.2 and $27.8 \mathrm{~nm}$ for Figure 5B, and $15.4 \mathrm{~nm}$ and $17.5 \mathrm{~nm}$ for Figure 5C). This supports the presence of highly anisotropic clusters such as (almost) linear ones. In Tables 2 and 3, we have reported the values of $r_{H}$ deduced from model (II) and (III) by using the coefficients given in Table 1 and for different numbers $n$ of NPs composing the aggregates. For each situation (Figures 5B and 5C) we obtain consistent values of $r_{H} \approx r_{H}^{0}=10 \mathrm{~nm}$ for a well 
determined $n$ which is moreover the same for both models. This unambiguously enables to analyze the $\mathrm{ACF}$ of Figure $5 \mathrm{~B}$ as resulting from the diffusion and rotation of linear aggregates composed of four NPs, and for Figure 5C to the diffusion and rotation of dimers. The former 4 bead case was also tested towards fitting by a tetrahedral model, for which the values $\alpha=0.89$ and $\beta=0.64$ are given in reference. ${ }^{43}$ The $r_{H}$ values as obtained are listed in Table 2 in the line specified with 4(T). As for the spherical model, the hypothesis of a tetrahedral cluster reveals inconsistent results, confirming that Figure 5B very likely corresponds to the observation of diffusion and rotation of a chain-like aggregate made up from 4 NPs.

In addition to these results, the ratios $R=0.75$ and $R=1.8$ as seen in Figures 5B and 5C need

Table 2: Values of $r_{H}$ calculated from the diffusion and rotation coefficients $D_{D}$ and $D_{R}$ extracted from the measured ACF shown in Figure 5B. The matching of the $r_{H}$ values as derived from $D_{D}$ and $D_{R}$ as well as the matching to the nominal value of the non-aggregated NPs occurs for $n=4$ and indicates that the ACF presented in Figure 5B originates from the diffusion of 4-NPs clusters. Calculation were made using Model (II) and (III) for different numbers $n$ of NPs in the aggregate. The line $4(T)$ stands for a tetrahedral model of 4 beads. The values noted $r_{H}(\alpha)$ are obtained from the diffusion coefficient $D_{D}$ while the values noted $r_{H}(\beta)$ are obtained from the rotational coefficient $D_{R}$.

\begin{tabular}{|c|c|c|c|c|}
\hline & \multicolumn{2}{|c|}{ Model (II) } & \multicolumn{2}{|c|}{ Model (III) } \\
\hline$n$ & 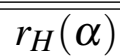 & $\overline{r_{H}(\beta)}$ & 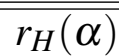 & $\overline{r_{H}(\beta)}$ \\
\hline 2 & 14.5 & 18.0 & 12.7 & 15.7 \\
\hline 3 & 11.7 & 13.3 & 10.7 & 12 \\
\hline 4 & 9.9 & 10.6 & 9.3 & 9.9 \\
\hline $4(T)$ & 18 & 24 & none & none \\
\hline 5 & 8.5 & 9.2 & 8.1 & 8.3 \\
\hline
\end{tabular}

some further comments. The value $R=0.75$ (Figure 5B) indicates that the rotational component represents $\sim 40 \%$ of the total amplitude of the ACF which is the threshold value given by Laurence et al. ${ }^{28}$ when measuring a single hot-spot, i.e. inspecting a unique preferential direction of scattering. In the present case, it can be estimated that one or two hot spots induce the rotational contribution, which is in accordance with the ACF revealing the diffusion of a chain of four NPs. The value $R=1.8$ (in Figure 5C) indicates that the rotational component represents $\sim 60 \%$ of the total amplitude of the ACF, which has been considered in literature as the signature of a unique 
Table 3: Values of $r_{H}$ calculated from the diffusion and rotation coefficients $D_{D}$ and $D_{R}$ extracted from the measured ACF shown in Figure 5C. The matching of the $r_{H}$ values as derived from $D_{D}$ and $D_{R}$ as well as the matching to the nominal value of the non-aggregated NPs occurs for $n=2$ and indicates that the ACF presented in Figure 5C originates from the diffusion of dimers. Calculation were made using Model (II) and (III) for different numbers $n$ of NPs in the aggregate. The values noted $r_{H}(\alpha)$ are obtained from the diffusion coefficient $D_{D}$ while the values noted $r_{H}(\beta)$ are obtained from the rotational coefficient $D_{R}$.

\begin{tabular}{|c|c|c|c|c|}
\hline & \multicolumn{2}{|c|}{ Model (II) } & \multicolumn{2}{|c|}{ Model (III) } \\
\hline$n$ & $\overline{r_{H}(\alpha)}$ & $r_{H}(\beta)$ & $r_{H}(\alpha)$ & $r_{H}(\beta)$ \\
\hline 2 & 11.3 & 11.3 & 9.9 & 9.9 \\
\hline 3 & 9.1 & 8.4 & 8.3 & 7.5 \\
\hline 4 & 7.7 & 6.7 & 7.2 & 6.2 \\
\hline 5 & 6.6 & 5.8 & 6.3 & 5.2 \\
\hline
\end{tabular}

hot-spot found in-between two particles. ${ }^{28}$ This result is also in accordance with the determination of a dimer in the situation of Figure 5C.

Finally, the last measurement presented in Figure 5D shows an ACF that hints towards the diffusion of non-aggregated NPs. This is revealed by a unique translational characteristic time $\tau_{D}=2.2$ ms which corresponds to an hydrodynamic radius $r_{H}=10.6 \approx r_{H}^{0} \mathrm{~nm}$ in full agreement with the nominal value measured from the initial isolated Ag NP solution by DLS. Moreover, the absence of a rotational contribution reinforces this result, as it is expected to have $R=0$ for isotropic scattering of molecules assumed to be uniformly adsorbed onto the spherical nanoparticle surface. Note that the ACF appears to be very noisy at shorter times and it cannot be completely ruled out that the rotational contribution is not measured owing to this noise. However, this noise, which stems from a weak SERS signal, is also in favor of the measure of the dynamics of unique NPs since the SERS enhancement is much smaller in this situation than in the case of molecules sitting in the gap between two NPs. Finally, optical observation confirms this presumption as the solution on which the ACF was measured remained yellow after adding MBA molecules, and its extinction spectrum did not present any supplementary absorption band in addition to that of the isolated NPs. These two observations both indicate the absence of any aggregation process in solution. It is thus possible to conclude that the ACF presented in Figure 5D stems from the fluctuations of the SERS signal of MBA molecules that are adsorbed onto isolated, non-aggregated NPs. The 
above results thus clearly show that the experiments have indeed enough sensitivity to detect and characterize even the very first stages of NP aggregation. It enables the clear distinction between the diffusion/rotation of single NPs, small aggregates up to larger cluster behavior.

\section{Conclusion}

In this paper we have demonstrated that SERS correlation spectroscopy is a very promising and powerful technique for in situ studies. It enables the quantitative sizing and characterization of metal colloidal solutions of SERS-active aggregates. The initial colloidal solution were monodisperse NPs of nominal hydrodynamic radius $r_{H}^{0}=10 \mathrm{~nm}$. By using an optical set-up especially optimized for such experiments, ${ }^{30}$ high quality ACFs of the SERS intensity of MBA molecules adsorbed on isolated or aggregated NPs were obtained. A quantitative exploitation of the ACFs was possible and allowed for identifying different stages of NP aggregation down to the scale of isolated NPs. The solutions measured in the present paper were very diluted such that there is statistically one or less aggregate at a time in the observation volume. The measured ACFs are consequently very likely to derived from single aggregates events, i.e from the SERS intensity fluctuations due to the entrance or exit of a single aggregate. However we do not know how many of such events occur during the measuring time. Work is under progress to access this parameter.

For large clusters of NPs, the SERS fluctuations are high and the signal is isotropic on average. Low noise ACFs were measured and showed the features expected from theory, i.e. the absence of a rotational characteristic time and a translational diffusion time corresponding to a hydrodynamic radius much larger than that of the isolated NPs. For smaller aggregates, the ACFs show more noise as the SERS intensity is lower. In spite of that, the ACFs corresponding to the diffusion of aggregates formed by only 2 to 4 NPs were successfully measured, and correctly analyzed by means of adequate theoretical models. In particular, the rotational diffusion time stemming from the anisotropic SERS scattering of chains of a few monomers was measured together with the translational diffusion time, and both values were associated, consistently one with each other, to the properties of multimers of a few NPs. Finally, the ACF of non-aggregated NPs was also deter- 
mined and fingerprinted by the absence of the rotational diffusion time and a translational diffusion time that corresponds to the hydrodynamic radius of the isolated NPs of the initial silver solution.

Further work is under progress to improve the determination of the mean number of NPs in solution, the size distribution of the aggregates and the sensibility of the chemical selectivity of our apparatus. Nevertheless, the present results seem already to pave the way for in situ (i.e in the reactive medium) characterization of functionalization processes on metal NPs as well as the study of aggregation processes. In particular, following the size of SERS-active clusters as a function of time would allow accessing their fractal dimension, which might be directly correlated to their SERS activity. Studying the influence of physical or chemical parameters such as the temperature or the $\mathrm{pH}$ of the solutions, on the formation of self-assembled NPs clusters could also be performed in the future and in a unique way by SERS correlation spectroscopy.

\section{Acknowledgement}

We wish to thank the Région Rhône Alpes for supporting this research via the grant CIBLE-RCS. LME acknowledges support by the German Science Foundation (DFG) through the Cluster of Excellence "Center of Advancing Electronics Dresden".

\section{References}

(1) Faraday, M. The Bakerian Lecture: Experimental Relations of Gold (and Other Metals) to Light. Phil. Trans. 1857,147, 145-181.

(2) Mie, G. Beiträge zur Optik Medien, Speziell Kolloidaler Metallösungen. Am. Physik 1908, $25,377-445$.

(3) Gersten, J. I. The Effect of Surface Roughness on Surface Enhanced Raman Scattering. J. Chem. Phys. 1980, 72, 5779-5780.

(4) Kneipp, K.; Moskovits, M.; Kneipp, H. (Eds.) Series: Topics in Applied Physics 2006, 103, 1-464. 
(5) Aravind, P. K.; Nitzan, A.; Metiu, H. The Interaction between Electromagnetic Resonances and its Role in Spectroscopic Studies of Molecules Adsorbed on Colloidal Particles or Metal Spheres. Surf. Sci 1981, 110, 189-204.

(6) Johansson, P.; Xu, H.; Käll, M. Surface-Enhanced Raman Scattering and Fluorescence Near Metal Nanoparticles. Phys. Rev. B 2005, 72, 035427-1-035427-17.

(7) Barbara, A.; Le Perchec, J.; Collin, S.; Sauvan, C.; Pelouard, J.L.; López-Ríos, T.; Quémerais, P. Generation and Control of Hot Spots on Commensurate Metallic Gratings. Optics Express 2008, 16, 19127-19135.

(8) Markel, V. A.; Shalaev, V. M.; Zhang, P.; Huynh, W.; Tay, L.; Haslett, T. L.; Moskovits, M. Near-Field Optical Spectroscopy of Individual Surface-Plasmon Modes in Colloid Clusters. Phys. Rev. B 1999, 59, 10903-10909.

(9) Nie, S.; Emory, S. R. by Surface-Enhanced Raman Scattering Probing Single Molecules and Single Nanoparticles. Science 1997, 275, 1102-1106.

(10) Kneipp, K.; Wang, Y.; Kneipp, H.; Perelman, L. T.; Itzkan, I.; Dasari, R. R.; Feld, M. S. Single Molecule Detection Using Surface-Enhanced Raman Scattering (SERS). Phys. Rev. Lett. 1997, 78, 1667-1670.

(11) Louit, G.; Asahi, T.; Tanaka, G.; Uwada, T.; Masuhara, H. Spectral and 3-Dimensional Tracking of Single Gold Nanoparticles in Living Cells Studied by Rayleigh Light Scattering Microscopy. J. Phys. Chem. C, 2009, 113, 11766-11772.

(12) Doane, T. L.; Burda, C. The Unique Role of Nanoparticles in Nanomedicine: Imaging, Drug Delivery and Therapy. Chem. Soc. Rev. 2012, 41, 2885-2911.

(13) Kim, H.; Kosuda, K. M.; Van Duyne, R. P.; Stair, P. C. Resonance Raman and Surface- and Tip-Enhanced Raman Spectroscopy Methods to Study Solid Catalysts and Heterogeneous Catalytic Reactions. Chem. Soc. Rev. 2010, 39, 4820-4844. 
(14) Zou, S.; Weaver, M. J. Surface-Enhanced Raman Scattering on Uniform Transition-Metal Films: Toward a Versatile Adsorbate Vibrational Strategy for Solid-Nonvacuum Interfaces? Anal. Chem. 1998, 70, 2387-2395.

(15) Fokas, C.; Deckert, V. Towards in situ Raman Microscopy of Single Catalytic Sites. Appl. Spectrosc. 2002, 56, 192-199.

(16) Nam, J.; Won, N.; Jin, H.; Chung, H.; Kim, S. pH-Induced Aggregation of Gold Nanoparticles for Photothermal Cancer Therapy.J. Am. Chem. Soc. 2009, 131, 13639-13645.

(17) Baffou, G.; Quidant, R.; García de Abajot, F. J. Nanoscale Control of Optical Heating in Complex Plasmonic Systems. ACS Nano 2010, 4, 709-716.

(18) Henry, A-I.; Bingham, J. M.; Ringe, E.; Marks, L. D.; Schatz, G. C.; Van Duyne, R. P. Correlated Structure and Optical Property Studies of Plasmonic Nanoparticles. J. Phys. Chem. C, 2011 115, 9291-9305.

(19) Loumaigne, M.; Praho, R.; Nutarelli, D.; Werts, M. H. V.; Débarre, A. Fluorescence Correlation Spectroscopy Reveals Strong Fluorescence Quenching of FITC Adducts on PEGylated Gold Nanoparticles in Water and the Presence of Fluorescent Aggregates of Desorbed Thiolate Ligands. Phys. Chem. Chem. Phys 2010, 12, 11004-11014.

(20) Paulo, P. M. R.; Gaiduk, A.; Kulzer, F.; Krens, S. F. G.; Spaink, H. P.; Schmidt, T.; Orrit, M. Photothermal Correlation Spectroscopy of Gold Nanoparticles in Solution. J. Phys. Chem. C 2009, 113, 11451-11457.

(21) Sebba, D. S.; Watson, D. A.; Nolan, J. P. High Throughput Single Nanoparticle Spectroscopy. ACS Nano 2009, 3, 1477-1484.

(22) Bingham, J. M.; Willets, K. A.; Shah, N. C.; Andrews, A. Q.; Van Duyne, R. P. Localized Surface Plasmon Resonance Imaging: Simultaneous Single Nanoparticle Spectroscopy and Diffusional Dynamics. J. Phys. Chem. C 2009, 113, 16839-16842. 
(23) Becker, J.; Schubert, O.; Sonnichsen, C. Gold Nanoparticle Growth Monitored in situ Using a Novel Fast Optical Single-Particle Spectroscopy Method. Nano Lett. 2007, 7, 1664-1669.

(24) Loumaigne, M.; Vasanthakumar, P.; Richard, A.; Débarre, A. Time-of-Flight Photon Spectroscopy: A Simple Scheme To Monitor Simultaneously Spectral and Temporal Fluctuations of Emission on Single Nanoparticles. ACS Nano 2012, 6, 10512-10523.

(25) Wark, A. W.; Stokes, R. J.; Darby, S. B.; Smith, W. E.; Graham, D. Dynamic Imaging Analysis of SERS-Active Nanoparticle Clusters in Suspension. J. Phys. Chem. C 2010, 114, 1811518120.

(26) O. Krichevsky, G. Bonnet, Fluorescence Correlation Spectroscopy: the Technique and its Applications. Rep. Prog. Phys. 2002, 65, 251-297.

(27) Eggeling, C.; Schaffer, J.; Seidel, C. A. M.; Korte, J.; Brehm, G.; Schneider, S.; Schrof, W. Homogeneity, Transport, and Signal Properties of Single Ag Particles Studied by SingleMolecule Surface-Enhanced Resonance Raman Scattering. J. of Phys. Chem. A 2001, 105, $3673-3679$.

(28) Laurence, T. A.; Braun, G.; Talley, C.; Schwartzberg, A.; Moskovits, M.; Reich, N.; Huser, T. Rapid, Solution-Based Characterization of Optimized SERS Nanoparticle Substrates. J. App. Chem. Soc. 2009, 131, 162-169.

(29) Laurence, T.; Braun, G. B.; Reich, N. O.; Moskovits, M. Robust SERS Enhancement Factor Statistics Using Rotational Correlation Spectroscopy. Nano Lett. 2012, 12, 2912-2917.

(30) Barbara, A.; Dubois, F.; Quémerais, P.; Eng, L. Non-Resonant and Non-Enhanced Raman Correlation Spectroscopy. Opt. Exp. 2013, 21, 15418-15429.

(31) Wustholz,K. L.; Henry, A-H.; McMahon, J. M.; Freeman, R. G.; Valley, N.; Piotti,M. E; Natan, M. J.; Schatz, G. C; Van Duyne, R. P. Structure-Activity Relationships in Gold 
Nanoparticle Dimers and Trimers for Surface-Enhanced Raman Spectroscopy. J. Am. Chem. Soc. 2010, 132, 10903-10910.

(32) Kask, P.; Piksarv, P.; Pooga, M.; Mets, Ü.; Lippmaa, E. Separation of the Rotational Contribution in Fluorescence Correlation Experiments. Biophys. J. 1989, 55, 213-220.

(33) Widengren, J.; Mets, Ü.; Rigler, R. Photodynamic Properties of Green Fluorescent Proteins Investigated by Fluorescence Correlation Spectroscopy. Chem. Phys. 1999, 250, 171-186.

(34) Aragón, S. R.; Pecora, R. Fluorescence Correlation Spectroscopy as a Probe of Molecular Dynamics. J. Chem. Phys. 1976, 64, 1791-1803.

(35) Ehrenberg, M.; Rigler, R. Rotational Brownian Motion and Fluorescence Intensity Fluctuations. Chem. Phys. 1974, 4, 390-401.

(36) Schaefer, D.W. Dynamics of Number Fluctuations: Motile Microorganisms. Science 1973, $180,1293-1295$.

(37) Thompson, N. In Topics in Fluorescence Spectroscopy, Volume I: Techniques, Ed. Joseph R. Lakowicz, Plenum Press, New York 1991.

(38) Gouÿ, L. Note sur le Mouvement Brownien J. de Phys. 1888, 7, 561-563.

(39) Einstein, A. On the Movement of Small Particules Suspended in Stationary Liquids Required by the Molecular-Kinetic Theory of Heat. Ann. d. Phys. 1905, 17, 549-560.

(40) Perrin, F. Mouvement Brownien d'un Ellipsoïde (I). Dispersion diélectrique pour des molécules Ellipsoïdales. J. de Phys. 1934, 10, 497-511.

(41) Bloomfield, V. A. Hydrodynamic Studies of Structure of Biological Macromolecules. Science 1968, $161,1212-1219$.

(42) Riseman, J.; Kirkwood, J. G. The Intrinsic Viscosity, Translational and Rotatory Diffusion Constants of RodLike Macromolecules in Solution. J. Chem. Phys. 1950, 18, 512-516. 
(43) García de la Torre, J.; del Rio Echenique, G.; Ortega, A. Improved Calculation of Rotational Diffusion and Intrinsic Viscosity of Bead Models for Macromolecules and Nanoparticles. $J$. Phys. Chem. B 2007, 111, 955-961.

(44) Adamczyk, Z.; Sadlej, K.; Wajnryb, E.; Ekiel-Jezewska, M. L.; Warszyński, P. Hydrodynamic Radii and Diffusion Coefficients of Particle Aggregates Derived from the Bead Model. J. Colloid Interface Sci. 2010, 347, 192-201.

(45) Lattuada, M.; Wu, H.; Morbidelli, M. Hydrodynamic Radius of Fractal Clusters. J. Colloid Interface Sci. 2003, 268, 96-105.

(46) Tirado, M. M.; López Martínez, C.; García de la Torre, J. Comparison of Theories for the Translational and Rotational Diffusion Coefficients of Rodlike Macromolecules. Application to Short DNA Fragments J. Chem. Phys. 1984, 81, 2047-2052.

(47) Creighton, J. A.; Blatchford, C. G.; Albrecht, M. G. Plasma Resonance Enhancement of Raman Scattering by Pyridine Adsorbed on Silver or Gold Sol Particles of Size Comparable to the Excitation Wavelength. J. Chem. Soc., Faraday Trans. II 1979, 75, 790-798.

(48) Lee, P. C.; Meisel, D. Adsorption and Surface-Enhanced Raman of Dyes on Silver and Gold Sols. J. Phys. Chem. 1982, 86, 3391-3395.

(49) Mulfinger, L.; Solomon, S. D.; Bahadory, M.; Jeyarajasingam, A. V.; Rutkowsky, S. A.; Boritz, C. Synthesis and Study of Silver Nanoparticles. J. of Chem. Edu. 2007, 84, 322-325.

(50) Yu, Y.; Handa, S.; Yajima, T.; Futamata, M. Flocculation of Ag Nanoparticles Elucidating Adsorbed p-Mercaptobenzoic Acid by Surface Enhanced Raman Scattering. Chem. Phys. Lett. 2013, 560, 49-54.

(51) Michota, A; Bukowska, J. Surface-Enhanced Raman Scattering (SERS) of 4Mercaptobenzoic Acid on Silver and Gold Substrates. J. Raman Spectrosc. 2003, 34, 21-25. 
(52) Fleger, Y.; Mastai, Y.; Rosenbluh, M.; Dressler, D. H. Surface Enhanced Raman Spectroscopy of Aromatic Compounds on Silver Nanoclusters. Surf. Science 2009, 603, 788-793.

(53) Pockrand, I. Surface Plasma Oscillations at Silver Surfaces with Thin Transparent and Absorbing Coatings. Surf. Science 1978, 72, 577-588.

(54) Meakin, P. Diffusion-Controlled Aggregation on Two-Dimensional Square Lattices: Results from a New Cluster-Cluster Aggregation Model. Phys. rev. B 1984, 29, 2930-2942.

(55) Kätzel, U.; Vorbau, M.; Stintz, M.; Gottschalk-Gaudig, T.; Barthel, H. Dynamic Light Scattering for the Characterization of Polydisperse Fractal Systems: II.Relation between Structure and DLS Results. Part. Part. Syst. Charact. 2008, 25, 19-30. 

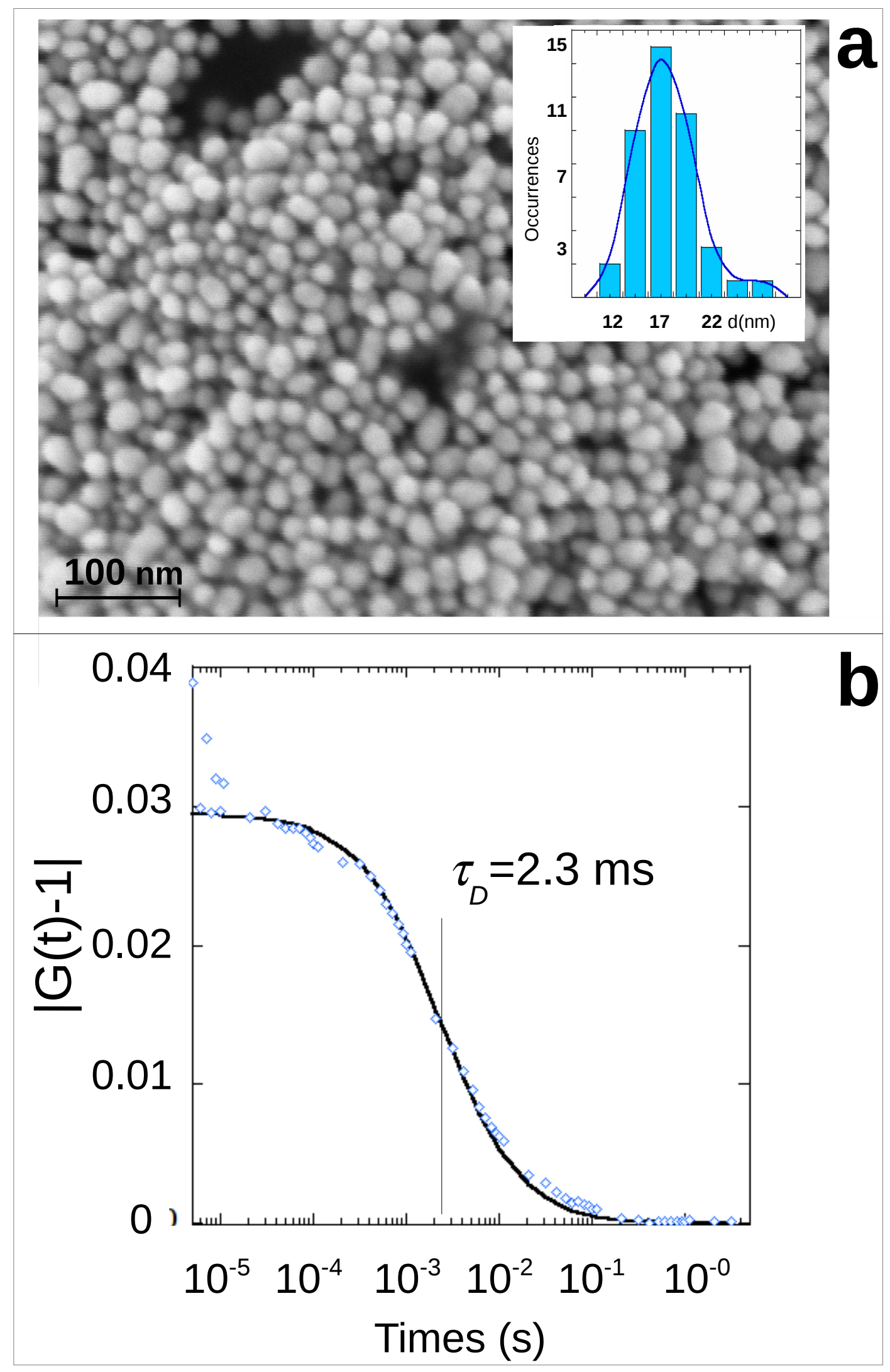

Figure 1: Example of SEM image of the NPs and distribution of their diameter obtained by statistical measurements of NPs (a). Example of DLS measurement (dots) of the silver solution performed using the optical set-up shown in Figure 2. Calculated data (line) are obtained using Eq. 2 and give a characteristic time $\tau_{D}=2.3 \mathrm{~ms}$ corresponding to an hydrodynamic radius $r_{H}^{0}=11 \mathrm{~nm}(\mathrm{~b})$ 


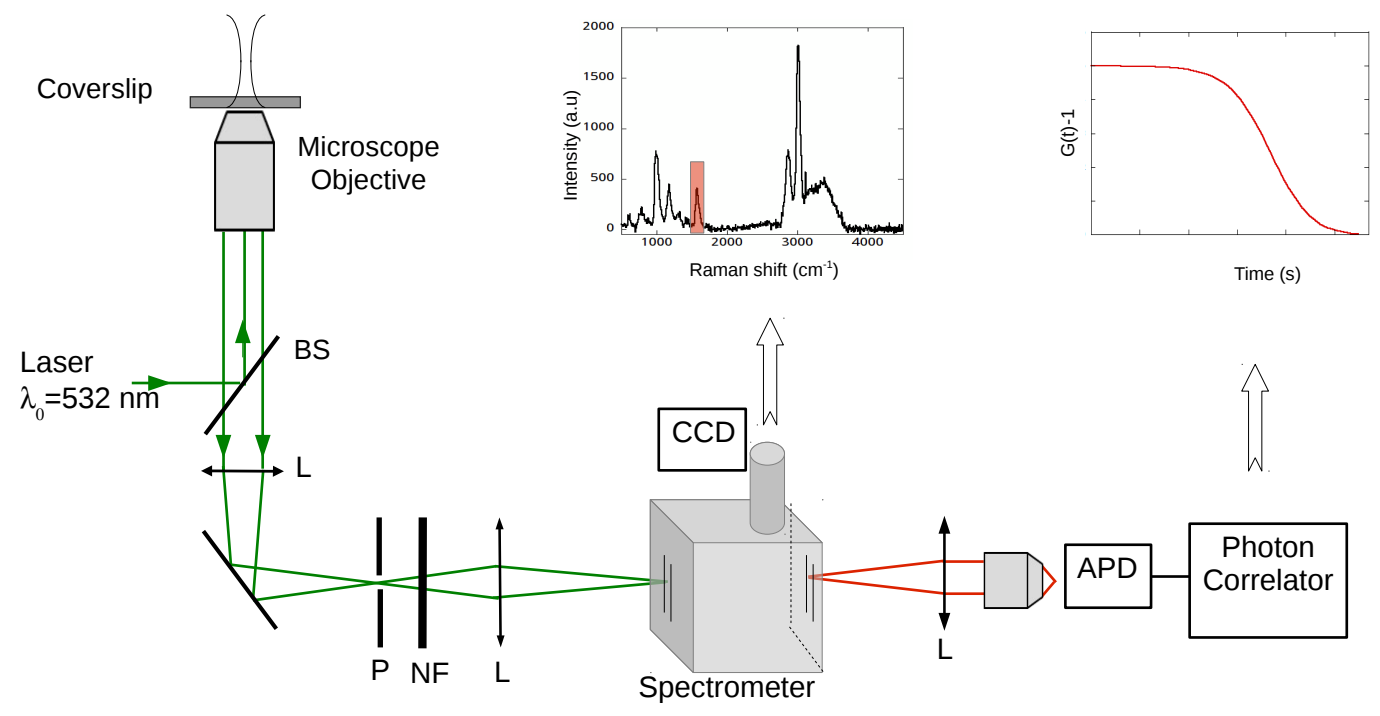

Figure 2: Scheme of the optical set-up. A $\lambda_{0}=532 \mathrm{~nm}$ laser is focused onto the solution through the microscope objective. The light backscattered by the particles inside the confocal volume is focused on a $300 \mu \mathrm{m}$ pinhole $(\mathrm{P})$. The Rayleigh scattering is rejected by a Notch filter (NF) while the Raman photons are focused at the entrance slits of a spectrometer. Two option are then possible: either realize Raman spectra using a cooled CCD camera or select a wavelength, usually corresponding the a Raman band, to exit the spectrometer. The outgoing signal is then focused on a avalanche photodiode (APD) coupled to a photon correlator that records the time-depend intensity $I_{\lambda}(t)$ and derives the autocorrelation functions $G(t)$. The letters BS stand for beam-splitter and L for lens. 

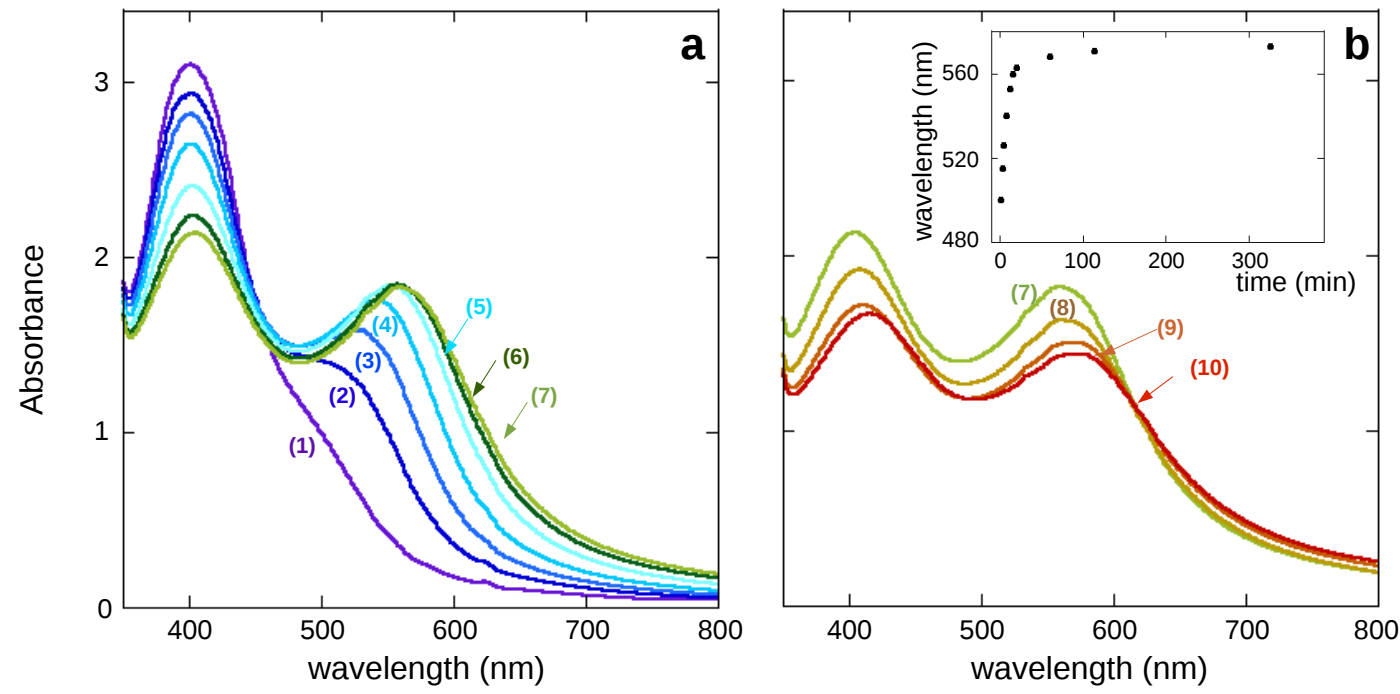

Figure 3: Absorbance spectra of a silver NPs solution in presence of $1.58 \mathrm{mM}$ of MBA recorded (a): 1 min (1), 3 min (2), 5 min (3), 8 min (4), $12 \min$ (5), 16 min (6) and 20 min (7), after adding the MBA to the Ag solution, and (b) at longer times: $60 \mathrm{~min}(8), 114 \mathrm{~min}(9)$ and $235 \mathrm{~min}$ (10) after adding the MBA to the Ag solution. Insert: Plotted is the wavelength of the longer-wavelengthabsorption peak as a function of time, displaying the kinetics and stabilization of the aggregation process. 


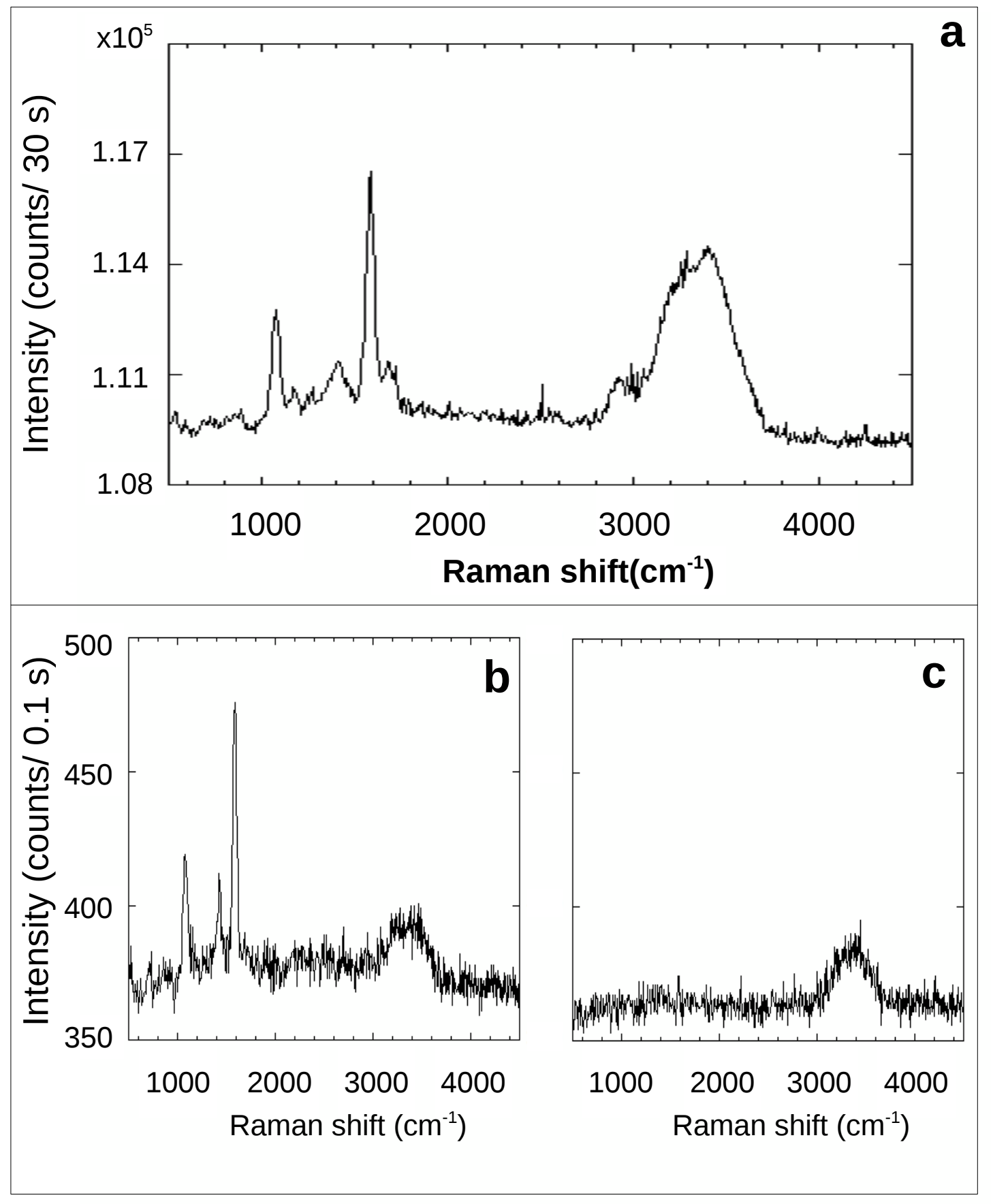

Figure 4: (a) Time-averaged Raman spectrum acquired for $30 \mathrm{~s}$ on a suspension of silver aggregates obtained by adding $C=1 \mathrm{mM}$ of MBA to the silver NP solution. (b),(c): Real-time Raman spectra with a $100 \mathrm{~ms}$ integration time showing the case when at least one SERS-active cluster was captured within the target volume (b), and the case when no such SERS-active cluster diffused through the observation volume (c). The latter thus represents the dark counts (background spectrum). 


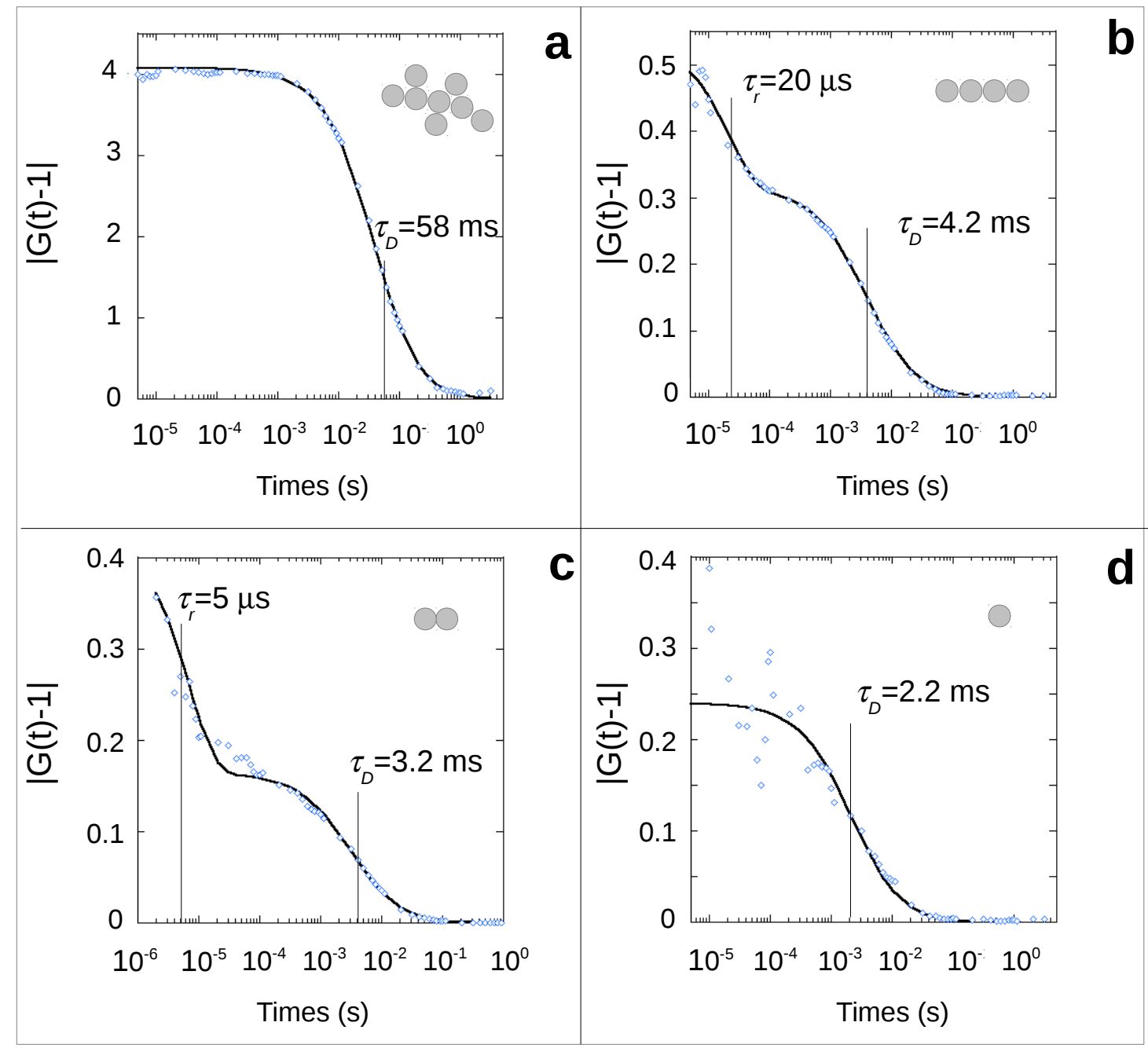

Figure 5: Experimental (dots) and calculated (line) ACFs of the SERS intensity of MBA molecules adsorbed on clusters in different aggregation stages. (a) ACF of a large cluster where the diffusion characteristic time $\tau_{D}$ corresponds to a hydrodynamic radius $r_{H}=280 \mathrm{~nm}$. (b) ACF of a cluster formed of 4 NPs as given by its diffusion characteristic time $\tau_{D}$ and the rotational contribution $\tau_{R}$ at short times (Table 2). (c) ACF of a dimer as given by its two characteristic times $\tau_{D}$ and $\tau_{R}$ (Table 3). (d) ACF of a single NP of radius $r_{H}=10 \mathrm{~nm}$. Note that as clusters grow (d-a), the intercept value $G(t=0)-1$ increases which is in accordance with the expected decrease of the average number of cluster $\langle N\rangle$. 


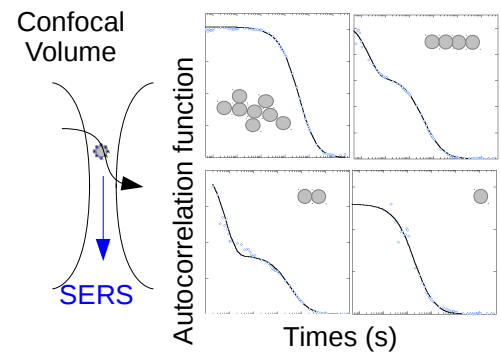

